\title{
Kompetenzen von Führungskräften und Coaches
}

\section{Astrid Schreyögg}

Online publiziert: 21. Oktober 2014

(C) Springer Fachmedien Wiesbaden 2014

„Kompetenz“ ist zwar heute ein zentraler Begriff in Fort- und Weiterbildungen, die meisten, die diesen Begriff verwenden, können aber nicht sagen, was sie genau darunter verstehen. Etymologisch entstammt er dem Lateinischen „competere“. Schon das ist aber durchaus mehrdeutig: Es meint zum einen ,fähig sein für etwas“. Es meint aber auch „,zugestehen, zukommen“. So können wir sagen: „Eine Peron ist in diesem oder jenem Sinn befähigt.“ WWir können aber auch sagen: „Eine Person ist zuständig für diesen oder jenen Bereich.“ Ohne hier in definitorische Tiefen abzugleiten, lässt sich bei einem Blick in sozialwissenschaftliche Lexika feststellen, dass wir es mit einem Containerbegriff zu tun haben, der über einen erheblichen Hof an Bedeutungen verfügt (vgl. Steinke 2015). So wird jeder, der sich mit Kompetenzen befasst, gar über sie schreibt, erläutern müssen, was er mit diesem Begriff verbindet. Das ist auch sinnvoll, denn in manchen Bereichen, wie wir sie hier thematisieren wollen, bei Führungskräften und bei Coaches, wird dieser Begriff besonders oft bemüht. Jeder Klient fragt sich nämlich, ob ,sein“ Coach auch ausreichend kompetent ist, mit ihm zu arbeiten. Und jeder Coach möchte der zu beratenden Führungskraft zu einem „Zugewinn an Kompetenzen“ verhelfen. Außerdem muss der Coach ausloten, für welchen Arbeitsbereich sein Klient zuständig bzw. kompetent ist. Im vorliegenden Heft haben wir zu diesen beiden Themenkomplexen, Kompetenzen von Coaches und Führungskräften, einige Aufsätze zusammengetragen.

Im ersten Beitrag beschäftigen sich Melanie Hasenbein und Daniela Riess-Beger mit einer kombinierten Fragestellung: Wie und ob Coaching-Kompetenzen auch für Führungskräfte nützlich sind. Auf der Basis von qualitativen Interviews kommen sie zu dem Ergebnis, dass manche Meta-Kompetenzen, also übergreifende Kompetenzen von Coaches, auch für Führungskräfte durchaus relevant sind. Der zweite Beitrag von Fabienne Theis und Anna Bergstermann enthält ein „Verfahren der Kompeten-

Dr. A. Schreyögg ( $\bowtie)$

Breisgauer Str. 29,

14129 Berlin, Deutschland

E-Mail: info@schreyoegg.de 
zenbilanz". Dabei handelt es sich ebenfalls um eine innovative Fragestellung: Welche Kompetenzen bringen Berufstätige in ein Studium mit, wie lassen sich diese Kompetenzen erfassen, und sind die Betreffenden selbst in der Lage, diese Kompetenzen zu würdigen? Im nachfolgenden Beitrag von Alexandra Michel, Carolin Merz, Andreas Frey und Karlheinz Sonntag geht es um die Entwicklung eines Kompetenzmodells für das Führungskräfte-Coaching. Die Autor/innen wollten ein empirisch fundiertes Kompetenzmodell entwerfen. Sie stützten sich dafür neben einer breiten Literaturrecherche auf eine Vielzahl von Experteninterviews. Kai Pfitzner geht in seinem Beitrag noch einen Schritt weiter und thematisiert die Steigerung der Performance, also der Leistungssteigerung durch Coaching. Da auch der Leistungsbegriff oft unklar bleibt, schlägt er eine Differenzierung in verschiedene Schritte vor, um die Begrifflichkeit und die Messung der Leistungssteigerung zu verbessern. Im fünften Fachbeitrag fragt Franziska Misch, ob Coaching von Führungskräften in der Altenpflege, also in einem sehr kräftezehrenden Arbeitsbereich, gesundheitsfördernde Effekte aufweist. Die Autorin beschreibt zunächst die speziellen Belastungsfaktoren, um sodann zu zeigen, dass die Gesundheit aller Beteiligten durch Coaching der jeweiligen Führungskräfte tatsächlich gefördert werden kann.

In einem Praxisbericht untersucht Carmen Wolf anhand einer Online-Befragung die Wirkungen der „Musterzustandsänderung“ im Kontext eines Coaching-Lehrgangs. In der Rubrik „Diskurse“ bieten wir zwei Beiträge: Uwe Böning und Frank Strikker beschäftigen sich mit der Frage, ob Coaching auf gesellschaftliche Entwicklungen nur reagieren soll, oder ob Coaching, zumal als Beratungsform für Entscheidungsträger, nicht auch als Impulsgeber für gesellschaftliche Innovationen fungieren kann. Der zweite Diskurs von Klaus Schenck befasst sich mit der Sprache im Coaching und ihren oft subtilen Wirkungen, die es sich bewusst zu machen lohnt. Der Autor macht darauf aufmerksam, dass besonders im Zusammenhang mit „Burnout“ metaphorische Sprachformen im Sinne von „Firewall“ oder „Lös(ch)wasser“ eine besondere Bedeutung haben.

\section{Literatur}

Steinke, I. (2015). Kompetenzanforderungen an Coaches. In A. Schreyögg \& C. Schmidt-Lellek (Hrsg.), Die Professionalisierung von Coaching. Ein Lesebuch für den Coach. Wiesbaden: Springer VS (im Druck). 